\title{
Rede Mãe Paranaense: assistência ao pré-natal entre mulheres nos extremos de idade
}

\section{Rede Mãe Paranaense: prenatal care among women at the age extremes}

Andressa Larissa Dias Müller de Souza' ${ }^{1}$, Adriana Zilly², Alexandrina Aparecida Maciel Cardelli ${ }^{3}$, Isabela Fernanda Larios Fracarolli ${ }^{4}$, Rosângela Aparecida Pimenta Ferrari ${ }^{5}$

1. ORCID: https://orcid.org/0000-0001-8582-5615. Enfermeira. Mestra em Enfermagem. Universidade Estadual de Londrina (UEL), Londrina, PR, Brasil. E-mail: andressadmuller@ gmail.com.

2. ORCID: https://orcid.org/0000-0002-8714-8205. Enfermeira. Doutora em Ciências Biológicas. Universidade Estadual do Oeste do Paraná (UNIOESTE), Foz do Iguaçu, PR, Brasil. E-mail: aazilly@hotmail.com.

3. ORCID: https://orcid.org/0000-0002-0222-8821.Enfermeira. Doutora em Saúde Pública. Universidade Estadual de Londrina (UEL), Londrina, PR, Brasil. E-mail: macielalexandrina@ gmail.com.

4. ORCID: http://orcid.org/0000-0003-3180-328X. Enfermeira. Especialista. Universidade Estadual de Londrina (UEL), Londrina, PR, Brasil. E-mail: isabela_larios@hotmail.com.

5. ORCID: http://orcid.org/0000-0003-0157-7461. Enfermeira. Doutora em Enfermagem, Orientadora. Universidade Estadual de Londrina (UEL), Londrina, PR, Brasil. E-mail: ropimentaferrari@uel.br.

CONTATO: Autor correspondente: Andressa Larissa Dias Müller de Souza | Endereço: Rua Florianópolis, 262, Alto da Glória, Gôiania, Goiás, Brasil, CEP 74815-770. Telefone: (62) 999665480. E-mail: andressadmuller@gmail.com

RESUMO Para garantir assistência de qualidade a gestante e ao neonato, o estado do Paraná, conta com a Rede Mãe Paranaense. Sendo assim, o estudo objetivou analisar a assistência pré-natal entre mulheres de extremo de idade, atendidas pela Rede, realizado em duas Regionais de Saúde, uma fronteiriça e outra no interior do Estado, entre julho de 2017 a fevereiro de 2018. Trata-se de um estudo quantitativo e transversal, os dados foram co- 
letados no prontuário, na Carteira de Saúde da gestante e entrevista. Os resultados revelaram que mulheres $\geq 35$ anos foram mais classificadas como alto risco gestacional, por antecedentes clínicos, como doenças hipertensivas $(p<0,004)$, entre as $\leq 18$ anos ocorreu menor adesão ao pré-natal $(p<0,012)$. A regional fronteiriça apresentou menos acompanhamento efetivo de pré-natal, também realizaram menos exames $(p<0,020)$. Concluindo-se que ocorreram divergências na assistência pré-natal entre os grupos etários, como também entre as regionais de saúde.

DESCRITORES: Avaliação de programas e projetos de saúde. Idade materna. Cuidado pré-natal. Saúde na fronteira.

ABSTRACT To ensure quality care for pregnant women and newborns, the state of Paraná has the Rede Mãe Paranaense. Therefore, this study aimed to analyze prenatal care among extremely age women, assisted by the Rede, held in two Health Regional Offices, one on the border and another in the interior of the state, between July 2017 and February 2018. This is a quantitative and cross-sectional study; data were collected from the medical record, from the pregnant woman's Health Card and from an interview. The results revealed that women aged $\geq 35$ years were more classified as having high gestational risk due to clinical history such as hypertensive diseases $(p<0.004)$, among those aged $\leq 18$ years, there was less adherence to prenatal care $(p<0.012)$. The regional border showed less effective prenatal care, also performed fewer tests $(p<0.020)$. In conclusion, there were divergences in prenatal care between age groups, as well as between regional health groups.

DESCRIPTORS: Health programs and projects evaluation. Maternal age. Prenatal care. Border health. 


\section{INTRODUÇÃO}

$\Delta$ idade materna é considerada um marcador para avaliação do risco gestacional, o Ministério da Saúde (MS) estabelece que idade maior que 35 anos e idade menor que 15 anos ou menarca há menos de 2 anos são características individuais que predispões a gestação em situação de risco ${ }^{1}$. Ainda relata que a adolescência em si, não é fator de risco para a gestação, mas existe possibilidade de risco psicossocial, como a imaturidade emocional, desestruturação familiar, situação econômica e baixa escolaridade. Em contrapartida o risco gestacional para mulheres de idade avançada está associado a maior predisposição à comorbidades preexistentes do período gravídico como também ao aumento de desfechos maternos graves quando comparadas com mulheres de 20 a 24 anos $^{2-3}$.

Com o intuito de garantir assistência de qualidade a gestante e ao neonato, o governo brasileiro criou em 2011, o programa Rede Cegonha, uma estratégia do MS pautada na inserção de recursos que assegurem as gestantes o direito ao planejamento reprodutivo, atendimento humanizado durante o processo de gravidez, parto e puerpério. Garantam a criança segurança no nascimento como também acompanhamento no processo de crescimento e desenvolvimento 4 .

Voltado para a responsabilidade estadual no acolhimento da gestante, o estado do Paraná, conta com a Rede Mãe Paranaense que tem como prioridade a inserção precoce da gestante no atendimento pré-natal, oferecendo ambulatório para pacientes de risco, garantia de parto hospitalar conforme a classificação do risco gestacional. Sendo considerado gestação de alto risco mulheres que apresentam hipertensão arterial, cardiopatias, pneumopatias, nefropatias em tratamento, diabetes, hipertireoidismo, hipotireoidismo, epilepsia, hemopatias (exceto anemia leve e moderada que são consideradas fisiológica da gestação), doenças infecciosas, doenças autoimunes, cirurgia útero/vaginal prévia, neoplasias, obesidade mórbida, cirurgia bariátrica realizada a menos de dois anos, psicose e depressão grave, dependência de drogas lícitas e ilícitas ${ }^{5}$.

Doenças prévias à gestação são, frequentemente mais encontradas, entre mulheres em idade avançada. Facilmente elas apresentam condições clínicas comuns, que dificultam o período gestacional, como hipertensão, diabetes mellitus, doenças cardiovasculares, obesidade, pré-eclâmpsia, parto tardio espontâneo e cesariana ${ }^{6}$.

O cuidado pré-natal é fundamental para garantir o desenvolvimento saudável da gravidez. Segundo uma pesquisa nacional, no Brasil a assistência pré-natal, independente da característica materna, foi superior a $90 \%$, mas a cobertura foi menor em residentes na Região Norte e indígenas ${ }^{7}$. 
Outra população vulnerável a uma menor cobertura pré-natal, são as "brasiguaias", esse termo de forma geral, refere-se as brasileiras que vivem no Paraguai. Mas na região da tríplice fronteira, Brasil, Paraguai e Argentina, também apresenta outros sentidos, tais como: brasileiro pobre que viveu no Paraguai e voltou para o Brasil; empresários brasileiros plantadores de soja no Paraguai; descendentes dos brasileiros que nasceram no Paraguai ${ }^{8}$.

As "brasiguaias" e outras gestantes moradoras nos países da tríplice fronteira, podem ser consideradas população vulnerável, pois realizam migração pendular em busca de serviços de saúde gratuito e de qualidade. Para receberem esse atendimento encontram formas extraoficiais de comprovar endereço na cidade brasileira. Essa população fronteiriça por medo de ter atendimento negado devido sua origem, ou por não dispor de condições financeiras para o deslocamento mensal que o pré-natal exige, geralmente procuram o serviço brasileiro em etapa avançada da gestação-10.

Diante do exposto, verificou-se a necessidade de analisar a assistência pré-natal ofertada pela Rede Mãe Paranaense entre mulheres de extremo de idade em Regionais de Saúde (RS), sendo uma localizada no interior do estado do Paraná $-17^{\text {a }}$ RS e a outra na região de fronteira $-9^{a} R S$.

\section{MÉTODO}

Trata-se de uma pesquisa quantitativa transversal realizada em duas Regionais de Saúde (RS) do Estado do Paraná, Sul do Brasil: $9^{a}$ RS região de fronteira com o Paraguai e a Argentina, $17^{\mathrm{a}} \mathrm{RS}$ localizada no interior do estado do Paraná e limite com o Estado de São Paulo. É um recorte do estudo multicêntrico intitulado: Rede Mãe Paranaense na perspectiva da usuária: o cuidado da mulher no pré-natal, parto, puerpério e da criança, realizado em três RS do estado, sendo $9^{a} \mathrm{RS}, 10^{\mathrm{a}} \mathrm{RS}$ e $17^{\mathrm{a}} \mathrm{RS}$, financiada pelo Conselho Nacional de Desenvolvimento Científico e Tecnológico (CNPq), chamada Universal 01/2016.

O Paraná tem 22 Regionais de Saúde e 04 macrorregionais: Leste, Oeste, Norte e Noroeste. Cada RS tem um município polo estabelecido de acordo com o desenvolvimento econômico e referência de serviços de alta complexidade, os polos também são municípios habilitados em Gestão Plena do Sistema Único de Saúde (SUS), como é o caso de Foz do Iguaçu polo da $9^{\mathrm{a} R S}$ e Londrina polo da $17^{\mathrm{a}} \mathrm{RS}{ }^{11}$.

A Rede Mãe Paranaense organizou os pontos de atenção hospitalar nas 22 regionais de saúde. Esses hospitais contam com leitos para atender as gestantes de acordo com o seu risco gestacional, leitos de UTI adulto, neonatal, pediátricos, unidade de cuidados complementares objetivando um atendimento de qualidade ${ }^{11}$. Portanto, esse estudo selecionou as maternidades que são estipuladas pela Rede, sendo duas para gestação de 
risco habitual $(\mathrm{RH})$ e uma que atende $\mathrm{RH}$ e alto risco $(\mathrm{AR})$ na $9^{\mathrm{a}} \mathrm{RS}$ totalizando atendimento de mulheres residentes nos nove municípios que compõem essa regional. Para a $17^{\mathrm{a}} \mathrm{RS}$, dos 21 municípios 16 foram contemplados, sendo quatro maternidades para gestação de RH e uma AR.

Realizou-se cálculo amostral proporcional com base no número de partos do ano de 2016, margem de erro da pesquisa de $5 \%$. O cálculo resultou em 409 mulheres da $9^{a} \mathrm{RS}$ e 464 da $17^{\text {a }}$ RS. Para o presente estudo, foram selecionadas as mulheres dos extremos de idade, até 18 anos 11 meses e 29 dias (adolescentes) e $\geq 35$ anos, totalizando 197 mulheres, sendo 87 da $9^{\mathrm{a}} \mathrm{RS}$ e 110 da $17^{\mathrm{a}} \mathrm{RS}$.

Os critérios de inclusão foram mulheres dos extremos de idade ( $\leq 18$ anos e $\geq 35$ anos) atendidas exclusivamente pelo SUS, em maternidades de referência para o parto na Rede, ter realizado o pré-natal no serviço de atenção primária a saúde e aceitar participar da pesquisa.

A coleta de dados foi realizada entre julho de 2017 e fevereiro de 2018 nas maternidades por meio de um instrumento previamente testado para extrair dados em prontuários da maternidade, Carteira de Saúde da Gestante e entrevista com a mulher/usuária para identificar informações não esclarecidos ou que não constavam nos documentos.

As variáveis desse estudo foram: situação conjugal, $\mathrm{n}^{\circ}$ de pessoas na casa, escolaridade, ocupação materna, renda familiar, $n^{\circ}$ de filhos, paridade, aborto, intervalo interpartal $<1$ ano, hábitos maternos (tabaco, álcool e drogas), antecedentes clínicos, método contraceptivo, classificação do risco gestacional (habitual, intermediário e alto), início do pré-natal, $n^{\circ}$ de consultas pré-natal, presença de ultrassonografia (USG) no $1^{\circ}, 2^{\circ}$ e $3^{\circ}$ trimestre de gestação, USG morfológica, exames laboratoriais no pré-natal por trimestre, exame das mamas, coleta de citologia oncótica, vacinação (antitetânica, anti-hepatite B e gripe H1N1), suplementação com ácido fólico e sulfato ferroso, desenvolvimento de doença durante a gestação, intercorrência gestacional, consulta odontológica, participação em grupos de pré-natal, fonte de informações na gestação, desejo em amamentar e sentimento de desrespeito durante o pré-natal. Foram classificadas conforme os indicadores da Rede para cada grupo de assistência pré-natal e comparada aos extremos de idade materna e RS.

Os dados foram inseridos e analisados no SSPS ${ }^{\circledR}$ - Statistical Package for the Social Sciences, por meio desse programa foi possível realizar as análises estatísticas, assumindo-se um nível de significância de $p<0,05$, aplicou-se o teste qui-quadrado e nas variáveis com valor $p<0,020$, realizou-se regressão logística binária e multinominal. Dessa maneira, foi possível identificar o risco relativo associado ao evento, sendo considerada como variável independente a idade materna e Regional de Saúde.

A pesquisa foi autorizada pela Secretaria de Saúde do Estado do Paraná, pelas Diretorias das Regionais de Saúde e aprovada pelo Comitê de Ética da Universidade Esta- 
dual de Londrina (CEP/UEL), sob o parecer $\mathrm{n}^{\circ}$ 2.053.304, de 09 de maio de 2017, CAAE: 67574517.1.1001.5231.

\section{RESULTADOS}

Totalizaram-se 197 puérperas nas RS, que compreendiam os extremos de idade materna. A média de idade do grupo de adolescentes em ambas as RS foi de 17 anos e 38 anos no grupo de mulheres $\geq 35$ anos. Os grupos etários por regional foram, na $9^{a} \mathrm{RS}$ $48,3 \% \leq 18$ anos e $51,7 \% \geq 35$ anos e, na $17^{\text {a }}$ RS, $58,2 \% \leq 18$ anos e $41,8 \% \geq 35$ anos.

As características socioeconômicas e demográficas maternas (Tabela 1) revelaram que a maioria das mulheres, em ambos os grupos etários e RS, tinham companheiro. A maior escolaridade foi mais frequente entre as mulheres $\geq 35$ anos $(p=0,004)$, sendo $71,1 \%$ na $9^{a}$ RS e $68,9 \%$ na $17^{\mathrm{a}} \mathrm{RS}$. A ocupação não remunerada foi mais evidente entre as adolescentes $(p=0,000)$ sendo $83,3 \%$ na $9^{\text {a }}$ RS e $98,4 \%$ na $17^{\text {a }} \mathrm{RS}$, como também a renda familiar menor $(p=0,000) 54,8 \%$ e $54,7 \%$, respectivamente.

Os antecedentes gestacionais, revelaram que, a maior parte das adolescentes eram primíparas $(p=0,000) 90,5 \%$ na $9^{\text {a }} \mathrm{RS}$ e, $84,4 \%$ na $17^{\mathrm{a}} \mathrm{RS}$. O histórico de aborto foi mais frequente entre as mulheres $\geq 35$ anos e, o intervalo interpartal $<1$ ano, entre as adolescentes $(p=0,000)$. Quanto aos hábitos maternos o tabagismo não apresentou relevância estatística entre os extremos de idade, mas fumar narguilé foi mais presente entre as adolescentes $(p=0,003)$, sendo $11,9 \%$ na $9^{a}$ RS. A presença de antecedentes clínicos foi mais evidente entre as mulheres acima de 35 anos de idade $(p=0,004)$, sendo $26,7 \%$ na $9^{a} \mathrm{RS}$ e $32,6 \%$ na $17^{\mathrm{a}} \mathrm{RS}$, com relevância as doenças hipertensivas $(p=0,002)$.

Tabela 1. Condições socioeconômicas e demográficas, antecedentes clínicos e gestacionais, conforme a faixa etária materna e Regional de Saúde (9ª e 17ª), Paraná, 2018.

\begin{tabular}{|c|c|c|c|c|}
\hline \multirow{4}{*}{ Variáveis } & \multicolumn{2}{|c|}{ 9A RS } & \multicolumn{2}{|c|}{$17 \stackrel{\text { RS }}{ }$} \\
\hline & $\leq 18$ anos & $\geq 35$ anos & $\leq 18$ anos & $\geq 35$ anos \\
\hline & n (\%) & n (\%) & n (\%) & n (\%) \\
\hline & $42(100,0)$ & $45(100,0)$ & $64(100,0)$ & $46(100,0)$ \\
\hline \multicolumn{5}{|l|}{ Situação conjugal } \\
\hline Com companheiro & $34(81,0)$ & $39(86,7)$ & $48(77,4)$ & $40(87,0)$ \\
\hline Sem companheiro & $8(19,0)$ & $6(13,3)$ & $14(22,6)$ & $6(13,0)$ \\
\hline \multicolumn{5}{|l|}{ № de pessoas na casa } \\
\hline$\leq 4$ & $27(64,3)$ & $23(51,1)$ & $44(68,8)$ & $32(69,6)$ \\
\hline$>4$ & $15(35,7)$ & $22(48,9)$ & $20(31,2)$ & $14(30,4)$ \\
\hline \multicolumn{5}{|l|}{ Escolaridade em anos* } \\
\hline$\leq 8$ & $22(52,4)$ & $13(28,9)$ & $31(48,4)$ & $14(31,1)$ \\
\hline$>8$ & $20(47,6)$ & $32(71,1)$ & $33(51,6)$ & $31(68,9)$ \\
\hline
\end{tabular}




\begin{tabular}{|c|c|c|c|c|}
\hline \multicolumn{5}{|l|}{ Ocupação materna* } \\
\hline Remunerada & $7(16,7)$ & $29(64,4)$ & $1(1,6)$ & $32(69,6)$ \\
\hline Não remunerada & $35(83,3)$ & $16(35,6)$ & $61(98,4)$ & $14(30,4)$ \\
\hline \multicolumn{5}{|l|}{ Renda familiar* } \\
\hline$<\mathrm{R} \$ 1405,00$ & $23(54,8)$ & $14(31,1)$ & $35(54,7)$ & $10(21,7)$ \\
\hline$\geq \mathrm{R} \$ 1405,00$ & $19(45,2)$ & $31(68,9)$ & $29(45,3)$ & $36(78,3)$ \\
\hline \multicolumn{5}{|l|}{ № de filhos* } \\
\hline Um & $39(92,9)$ & $7(15,6)$ & $59(92,2)$ & $11(23,9)$ \\
\hline Dois & $2(4,8)$ & $14(31,1)$ & $5(7,8)$ & $15(32,6)$ \\
\hline Três ou mais & $1(2,4)$ & $24(53,3)$ & 0 & $20(43,5)$ \\
\hline \multicolumn{5}{|l|}{ Paridade* } \\
\hline Primípara & $38(90,5)$ & $3(6,7)$ & $54(84,4)$ & $7(15,2)$ \\
\hline Multípara & $4(9,5)$ & $42(93,3)$ & $10(15,6)$ & $39(84,8)$ \\
\hline \multicolumn{5}{|l|}{ Hábitos Materno } \\
\hline Cigarro & $8(19,0)$ & $9(20,0)$ & $6(9,5)$ & $11(23,9)$ \\
\hline Narguile* & $5(11,9)$ & 0 & 0 & 0 \\
\hline Álcool & $1(2,4)$ & $3(6,7)$ & $2(3,2)$ & $2(4,3)$ \\
\hline Drogas & 0 & 0 & $1(1,6)$ & 0 \\
\hline Abandonou o consumo & $9(69,2)$ & $2(18,2)$ & $1(14,3)$ & $2(18,2)$ \\
\hline Diminuiu o consumo & $2(50,0)$ & $4(44,4)$ & $5(83,3)$ & $8(88,9)$ \\
\hline Antecedentes clínicos * & $4(9,5)$ & $12(26,7)$ & $10(15,6)$ & $15(32,6)$ \\
\hline Hipertensivo* & 0 & $3(6,8)$ & 0 & $3(6,5)$ \\
\hline Metabólico & $1(2,4)$ & $1(2,2)$ & 0 & $3(6,5)$ \\
\hline Infeccioso & $2(4,8)$ & $1(2,2)$ & $3(4,7)$ & $5(10,9)$ \\
\hline Respiratório & 0 & $1(2,2)$ & $6(9,4)$ & $1(2,2)$ \\
\hline Outros & $1(2,4)$ & $10(22,2)$ & $2(3,1)$ & $5(10,9)$ \\
\hline Aborto* & $2(4,8)$ & $11(24,4)$ & $2(4,3)$ & $10(23,3)$ \\
\hline Intervalo interpartal $<1$ ano* & $1(10,0)$ & $1(2,4)$ & $4(18,2)$ & $1(2,6)$ \\
\hline
\end{tabular}

Fonte: elaborado pelos autores (2018)

A assistência pré-natal conforme a faixa etária materna (Tabela 2) identifica que a não utilização de método contraceptivo foi frequente em ambas as faixas etárias, pouco mais entre as adolescentes, $61,9 \%$ na $9^{\text {a }}$ RS e $63,9 \%$ na $17^{\text {a }}$ RS.

A classificação do risco gestacional no pré-natal evidenciou que as mulheres acima de 35 anos foram mais classificadas como AR $(p=0,000) 42,9 \%$ na $9^{a}$ RS e $43,2 \%$ na $17^{\text {a }}$ RS. O início do pré-natal no primeiro trimestre ocorreu entre a maioria das mulheres sem relevância estatística nos extremos de idade, em contrapartida a maior adesão para $\geq 7$ consultas foi no grupo $\geq 35$ anos ( $p=0,011$ ), sendo $77,8 \%$ na $9^{\text {a }} \mathrm{RS}$ e $89,1 \%$ na $17^{\mathrm{a}} \mathrm{RS}$.

O exame das mamas e a coleta de citologia oncótica ocorreu em maior proporção entre aquelas com mais de 35 anos do que as adolescentes $(p<0,009)$. A cobertura vacinal, 
antitetânica e gripe (H1N1), foi acima de 95\% em ambos os extremos de idade materna e Regionais de Saúde. A utilização de ácido fólico e sulfato ferroso durante a gestação esteve mais presente entre as mulheres $\geq 35$ anos, assim como a prevalência de doença hipertensiva durante a gestação, $15,6 \%$ na $9^{\mathrm{a}} \mathrm{RS}$ e $17,4 \%$ na $17^{\mathrm{a}} \mathrm{RS}$.

As intercorrências na gestação não apresentaram relevância estatística comparando-se os extremos de idade materna. Mas, a fonte de informações que a mulher buscou durante a gestação obteve diferença, sendo mais evidente a internet entre as adolescentes $69 \%$ da $9^{\mathrm{a}} \mathrm{RS}$ e $72,3 \%$ da $17^{\mathrm{a}} \mathrm{RS}$ e, para as com mais de 35 anos o enfermeiro, $11,1 \%$ na $9^{a}$ RS e $20 \%$ na $17^{\text {a }}$ RS. O desejo de amamentar esteve presente em mais de $91 \%$ das mulheres em ambos os grupos etários.

Tabela 2. Assistência pré-natal conforme a faixa etária materna e Regional de Saúde $\left(9^{\mathrm{a}}\right.$ e $\left.17^{\mathrm{a}}\right)$, Paraná, 2018.

\begin{tabular}{|c|c|c|c|c|}
\hline \multirow[b]{2}{*}{ Variáveis } & \multicolumn{2}{|c|}{ 9A RS } & \multicolumn{2}{|c|}{ 17A RS } \\
\hline & $\begin{array}{c}\leq 18 \text { anos } \\
n(\%) \\
42(100,0)\end{array}$ & $\begin{array}{c}\geq 35 \text { anos } \\
n(\%) \\
45(100,0)\end{array}$ & $\begin{array}{c}\leq 18 \text { anos } \\
n(\%) \\
64(100,0)\end{array}$ & $\begin{array}{c}\geq 35 \text { anos } \\
n(\%) \\
46(100,0)\end{array}$ \\
\hline \multicolumn{5}{|l|}{ Método contraceptivo (MC) } \\
\hline Não usava & $26(61,9)$ & $20(44,4)$ & $39(63,9)$ & $23(50,0)$ \\
\hline Preservativo masculino & $3(7,1)$ & $8(17,8)$ & $9(14,8)$ & $5(10,9)$ \\
\hline Contraceptivo hormonal & $1331,0)$ & $17(37,8)$ & $13(21,3)$ & $18(39,1)$ \\
\hline \multicolumn{5}{|l|}{ Ausência do MC } \\
\hline Queria engravidar & $11(26,8)$ & $18(40,0)$ & $17(32,7)$ & $13(34,2)$ \\
\hline Falhou/esqueceu de usar & $14(34,1)$ & $16(35,6)$ & $14(26,9)$ & $14(36,8)$ \\
\hline \multicolumn{5}{|l|}{ Classificação do risco* } \\
\hline Risco habitual & $26(68,4)$ & $17(40,5)$ & $37(62,7)$ & $14(31,8)$ \\
\hline Risco intermediário & $8(21,1)$ & $7(16,7)$ & $13(22,0)$ & $11(25,0)$ \\
\hline Alto risco & $4(10,5)$ & $18(42,9)$ & $9(15,3)$ & $19(43,2)$ \\
\hline \multicolumn{5}{|l|}{ Início do pré-natal } \\
\hline$<14$ semanas & $20(54,1)$ & $24(66,7)$ & $51(79,7)$ & $38(82,6)$ \\
\hline $14-27$ semanas & $16(43,2)$ & $11(30,6)$ & $11(17,2)$ & $8(17,4)$ \\
\hline$>27$ semanas & $1(2,7)$ & $1(2,8)$ & $2(3,1)$ & 0 \\
\hline \multicolumn{5}{|l|}{ № de consultas pré-natal* } \\
\hline$\leq 6$ & $15(35,7)$ & $10(22,2)$ & $19(29,7)$ & $5(10,9)$ \\
\hline$\geq 7$ & $27(64,3)$ & $35(77,8)$ & $45(70,3)$ & $41(89,1)$ \\
\hline Doença na gestação & $11(26,2)$ & $11(24,4)$ & $27(42,2)$ & $23(50,0)$ \\
\hline Hipertensiva & $2(4,8)$ & $7(15,6)$ & $6(9,4)$ & $8(17,4)$ \\
\hline Metabólica & $3(7,1)$ & $1(2,2)$ & $5(7,8)$ & $10(21,7)$ \\
\hline Infecciosa & $4(9,5)$ & $4(8,9)$ & $13(20,3)$ & $8(17,4)$ \\
\hline
\end{tabular}




\begin{tabular}{lcccc} 
Intercorrência gestacional & $13(31,0)$ & $13(28,9)$ & $20(31,7)$ & $13(28,3)$ \\
$\quad$ Hipertensiva & 0 & $3(6,7)$ & $4(6,3)$ & $4(8,7)$ \\
Trabalho de parto prematuro & $1(2,4)$ & $1(2,3)$ & $2(3,2)$ & 0 \\
$\quad$ Sangramento & $5(12,2)$ & $7(15,6)$ & $3(4,8)$ & $2(4,3)$ \\
\hline Desejava amamentar & $40(95,2)$ & $44(97,8)$ & $59(92,2)$ & $42(91,3)$ \\
\hline Exame das mamas* & $17(41,5)$ & $22(51,2)$ & $18(34,6)$ & $25(64,1)$ \\
Coleta de citologia oncótica* & $19(45,2)$ & $16(35,6)$ & $28(44,4)$ & $32(72,7)$ \\
\hline Vacinação antitetânica (dTPa) & $40(95,2)$ & $42(95,5)$ & $61(98,4)$ & $44(97,8)$ \\
Vacinação anti-hepatite B & $35(83,3)$ & $36(81,8)$ & $62(98,4)$ & $42(93,3)$ \\
Vacinação de gripe (H1N1) & $38(95,0)$ & $42(97,7)$ & $60(95,2)$ & $41(97,6)$ \\
Usou ácido fólico* & $30(71,4)$ & $38(84,4)$ & $48(75,0)$ & $42(91,3)$ \\
\hline Usou sulfato ferroso & $37(88,1)$ & $40(88,9)$ & $52(81,2)$ & $43(95,6)$ \\
Consulta odontológica & $19(45,2)$ & $16(35,6)$ & $36(56,2)$ & $27(60,0)$ \\
\hline Desrespeitada no pré-natal & $2(4,8)$ & $4(8,9)$ & $1(1,6)$ & $4(8,7)$ \\
Participação grupos pré-natal & $6(14,3)$ & $6(13,3)$ & $18(29,0)$ & $4(8,9)$ \\
\hline Fonte informação na gestação & $32(76,2)$ & $25(55,6)$ & $42(65,6)$ & $27(58,7)$ \\
$\quad$ Fonte - internet* & $29(69,0)$ & $19(42,2)$ & $34(72,3)$ & $21(70,0)$ \\
Fonte - mãe/sogra & $12(28,6)$ & $2(4,4)$ & $16(35,6)$ & $7(23,3)$ \\
Fonte- enfermeiro & $2(4,8)$ & $5(11,1)$ & $1(2,2)$ & $6(20,0)$ \\
Fonte- médico & $5(11,9)$ & $7(15,6)$ & $11(23,9)$ & $9(30,0)$ \\
\hline
\end{tabular}

*Variáveis com valor de $p<0,05$

Fonte: elaborado pelos autores (2018)

Comparando a assistência pré-natal entre as RS (Tabela 3), identifica-se que na $17^{\text {a }}$ RS a mulher apresentou maior probabilidade de desenvolver alguma doença durante a gestação $(R R 0,40)$ do que as da $9^{a} R S$. Como também na $17^{a} R S$ as mulheres realizaram ao menos uma ultrassonografia (USG) obstétrica em cada trimestre de gestação (RR 0,36), USG morfológica (RR 0,37) e consulta com dentista (RR 0,49) do que as mulheres na $9^{a} R S$.

As gestantes atendidas na $9^{a} \mathrm{RS}$ tiveram maior risco para início tardio do cuidado pré-natal (RR 2,87). Poucas gestantes tiveram todos os exames laboratoriais, sendo que na $9^{a} R S$ a mulher teve mais risco para que não fossem soliacessos ( $R R$ 0,20). Também mais risco de esquema de vacinação incompleto da anti-hepatite $B(R R 0,18)$ e menores chances de coleta de citologia oncótica no pré-natal, ou em período $<1$ ano antes da gravidez (RR 0,50). 
Tabela 3. Regressão dos dados sobre a assistência pré-natal, conforme as Regionais de Saúde (9a e 17ạ), Paraná, 2018.

\begin{tabular}{|c|c|c|c|c|}
\hline \multirow[t]{3}{*}{ Variáveis } & \multicolumn{2}{|c|}{ Regional de Saúde } & \multirow{3}{*}{$\begin{array}{l}\text { Risco Relativo } \\
\text { (IC 95\%) }\end{array}$} & \multirow{3}{*}{ Valor $p$} \\
\hline & $\begin{array}{c}9 a \\
\text { n (\%) }\end{array}$ & $\begin{array}{c}17^{a} \\
n(\%)\end{array}$ & & \\
\hline & $87(100,0)$ & $110(100,0)$ & & \\
\hline \multicolumn{5}{|l|}{ Idade Materna } \\
\hline$\leq 18$ anos & $42(48,3)$ & $64(58,2)$ & & \\
\hline$\geq 35$ anos & $45(51,7)$ & $46(41,8)$ & $1,49(0,84-2,62)$ & 0,167 \\
\hline \multicolumn{5}{|l|}{ Intercorrência na gestação } \\
\hline Sangramento uterino & $12(14,0)$ & $5(4,6)$ & $3,37(1,14-9,98)$ & 0,028 \\
\hline \multicolumn{5}{|l|}{ Início do pré-natal } \\
\hline$<14$ semanas & $44(60,3)$ & $89(80,9)$ & & \\
\hline $14-27$ semanas & $27(37,0)$ & $19(17,30)$ & $2,87(1,44-5,72)$ & 0,003 \\
\hline$>27$ semanas & $2(2,7)$ & $2(1,8)$ & $2,02(0,27-14,8)$ & 0,488 \\
\hline Fez USG* nos três trimestres & $38(43,7)$ & $75(68,2)$ & $0,36(0,20-0,64)$ & 0,001 \\
\hline USG* morfológica & $41(48,8)$ & $71(71,7)$ & $0,37(0,20-0,69)$ & 0,002 \\
\hline USG* no 10 trimestre & $46(53,5)$ & $79(71,8)$ & $0,45(0,24-0,81)$ & 0,009 \\
\hline USG* no $2 \circ$ trimestre & $71(83,5)$ & $105(97,2)$ & $0,14(0,04-0,52)$ & 0,003 \\
\hline USG* no 3 o trimestre & $66(77,6)$ & $104(96,3)$ & $0,13(0,04-0,41)$ & 0,000 \\
\hline \multicolumn{5}{|l|}{ Exames laboratoriais RPM** } \\
\hline 10 Trimestre & $8(9,2)$ & $17(15,5)$ & $1,80(0,74-4,40)$ & 0,194 \\
\hline 20 Trimestre & $5(5,7)$ & $17(15,5)$ & $2,99(1,05-8,48)$ & 0,039 \\
\hline 3ㅇ Trimestre & $5(5,7)$ & $25(22,7)$ & $0,20(0,07-0,56)$ & 0,002 \\
\hline Doença durante gestação & $22(25,3)$ & $50(45,5)$ & $0,40(0,22-0,74)$ & 0,004 \\
\hline Vacinação anti-hepatite B & $71(82,6)$ & $104(96,3)$ & $0,18(0,05-0,57)$ & 0,004 \\
\hline Coleta de citologia oncótica & $34(39,1)$ & $60(56,1)$ & $0,50(0,28-0,89)$ & 0,019 \\
\hline Consulta odontológica & $35(40,2)$ & $63(57,8)$ & $0,49(0,27-0,87)$ & 0,015 \\
\hline
\end{tabular}

Fonte: elaborado pelos autores (2018)

\section{DISCUSSÃO}

A adolescência é uma etapa marcada por transformações biológicas, psíquicas e sociais, neste período, a gestação repercute com alterações psicossociais, como o abandono da escola e diminuição da convivência social ${ }^{2}$. Estudos ainda relatam redução das oportunidades de emprego, perpetuação da pobreza, aumento da discriminação, depressão e baixa autoestima. Também afirmam que a desvantagem socioeconômica e a baixa escolaridade são tanto antecedente quanto uma consequência da maternidade adolescente ${ }^{12,13}$. Neste estudo evidenciou-se que a condição de ocupação não 
remunerada, renda familiar menor e baixa escolaridade, ocorreu mais frequentemente entre as adolescentes.

Uma pesquisa americana relata que mulheres encorajadas a alcançar seus objetivos educacionais adiam a gestação ${ }^{14}$, já um estudo realizado na Tailândia com gestantes "mais velhas", relatam que quase $80 \%$ eram multíparas ${ }^{15}$. No presente estudo as mulheres $\geq 35$ anos apresentaram escolaridade mais elevada e multiparidade.

Neste estudo o histórico de aborto foi mais frequente entre as mulheres $\geq 35$ anos, diferentemente do que é relatado em um estudo americano, no qual cerca de $80 \%$ das gestações concebidas durante a adolescência não foram intencionais, corroborando para alta taxa de abortos nessa população ${ }^{13}$. Nos EUA, o aborto induzido pode ser realizado legalmente, em 2015, as taxas de aborto foram mais altas neste grupo etário do que nas mulheres acima de 25 anos $^{16}$.

No Brasil o aborto é vedado por lei, mas isso não extingue sua ocorrência, são praticados abortos clandestinos, inseguros e geralmente, o procedimento é realizado por pessoas não-habilitadas ou/e em ambiente inapropriado, elevando o aborto como uma das principais causas de morte materna, juntamente com distúrbios hipertensivos, hemorragia e sepse ${ }^{17-19}$.

No presente estudo o intervalo interpartal $<1$ ano ocorreu mais vezes entre adolescentes. Pesquisadores brasileiros evidenciaram em seu estudo que a repetição de gravidez na adolescência esteve fortemente associada a menor nível educacional e com maiores taxas de abandono escolar ${ }^{20}$. Pesquisa australiana demonstra que maior nível de escolaridade foi evidenciado como fator de proteção para chances de gravidez repetidas na adolescência ${ }^{19}$. Estudos citam que o uso de contracepção reversível de ação prolongada reduziu a repetição de gravidez entre as adolescentes ${ }^{12-13}$.

Embora o tabagismo não tenha apresentado relevância estatística, fumar narguilé ocorreu mais frequentemente entre as adolescentes na $9^{\text {a }} \mathrm{RS}$ do presente estudo, isso deve-se a forte influência árabe em Foz do Iguaçu, visto que nesta cidade encontra-se a segunda maior comunidade árabe do Brasil ${ }^{21}$. Um estudo realizado com adolescentes que residem em Foz do Iguaçu, revelou que $76 \%$ deles já utilizaram o narguilé pelo menos uma vez, a média de idade da experimentação foi entre 12 e 15 anos e, esse hábito, apresentou forte influência pela rede social de amizades ${ }^{22}$.

Pesquisadores australianos relatam em seu estudo, que as taxas do tabagismo na gravidez têm diminuído, mas que mães adolescentes têm maior probabilidade de usar álcool, drogas, "vaping" e narguilé, que embora os últimos são menos focalizados nas diretrizes, devem ser avaliados na gestação ${ }^{12}$. Ainda pesquisadores brasileiros apontam para a frequência de fumantes passivos na adolescência, $35,6 \%$ relataram ter pai, mãe ou irmãos fumantes e $47,6 \%$ amigos fumantes ${ }^{23}$. 
Gestantes com idade mais elevada apresentam taxas mais altas de antecedentes clínicos como hipertensão crônica, diabetes mellitus e disfunções da glândula tireoide ${ }^{15}$. Ao envelhecer a mulher passa por mudanças biológicas, o sistema vascular uterino vai diminuindo sua capacidade em se adaptar aos efeitos da gestação aumentando chance de desfechos perinatais adversos, como diabetes e hipertensão gestacional, pré-eclâmpsia e aborto ${ }^{6,15,24}$. Condições clínicas pré-existentes são relatadas pela Rede Mãe Paranaense como gravidez de alto risco ${ }^{5}$, o que foi constatado nesse estudo, visto que, as mulheres $\geq 35$ anos apresentaram mais antecedentes clínicos e foram classificadas quanto ao seu risco gestacional, como também doenças durante a gestação foram mais elevadas nessa faixa etária.

Fisiologicamente adolescentes grávidas apresentam risco de desenvolvimento de síndromes obstétricas, como a hipertensão gestacional e a pré-eclâmpsia ${ }^{25-26}$. No presente estudo adolescentes sem antecedentes clínicos desenvolveram hipertensão gestacional.

Adolescentes demonstram dificuldade em iniciar o pré-natal, implicando na detecção precoce de problemas comuns nessa população, como uso de álcool, drogas lícitas e ilícitas, infecções sexualmente transmissíveis ${ }^{12}$. A literatura relaciona a adolescente com a baixa adesão ao pré-natal, início tardio, participação em menos consultas associando-se com pré-natal inadequado ${ }^{27-28}$. Neste estudo, a participação em menos consultas de pré-natal que o preconizado pela Rede Mãe Paranaense, ocorreu mais frequentemente entre as adolescentes.

No Brasil, uma análise da assistência pré-natal oferecida às gestantes usuárias de serviços de saúde públicos e/ou privados no período entre 2011 e 2012, demonstrou que a cobertura foi de $98,7 \%$, sendo que, $75,8 \%$ das mulheres iniciaram o pré-natal antes da $16^{\text {a }}$ semana gestacional, $73,1 \%$ compareceram a seis ou mais consultas, sendo as Unidades Básicas de Saúde (89,6\%) principal local de realização do pré-natal ${ }^{7}$. Isto também foi evidenciado nesse estudo, pois o início ocorreu no primeiro trimestre entre as mulheres sem relevância estatística nos extremos de idade, embora a participação em no mínimo sete consultas pré-natal foi mais frequente entre as mulheres $\geq 35$ anos.

O exame das mamas e a coleta de citologia oncótica, conforme preconizado no pré-natal, realizou-se em menos da metade das mulheres desse estudo, maior proporção no grupo etário $\geq 35$ anos. Embora o diagnóstico do câncer durante a gravidez tenha ocorrência rara, a detecção precoce é fundamental para otimizar o resultado oncológico, mas infelizmente os sintomas relacionados ao câncer como fadiga, náusea, dor abdominal e perda de sangue vaginal, podem ser mal interpretados como sintomas fisiológicos relacionados à gravidez, reafirmando a importância dos exames de triagem durante a gestação ${ }^{29}$.

A anemia é encontrada em cerca de $50 \%$ das adolescentes grávidas, a suplementa- 
ção com ácido fólico e sulfato ferroso é indicada, porém o cumprimento adequado do cuidado pré-natal é um desafio, devido aos "maus" comportamentos de saúde entre as adolescentes ${ }^{13}$. Isto pode ser evidenciado nesse estudo, pois a suplementação durante a gestação foi maior entre as mulheres $\geq 35$ anos.

As Regionais de Saúde analisadas nesse estudo apresentam algumas características antagônicas, a $9^{a}$ RS compreende uma área urbana conhecida como tríplice fronteira, divisa entre Brasil, Paraguai e Argentina, diferentemente da $17^{\text {a }} \mathrm{RS}$ localizada no interior do estado do Paraná limite com São Paulo, maior região metropolitana do Brasil. Essa disparidade refletiu com indicadores ruins para a $9^{a} \mathrm{RS}$, visto que as mulheres da região fronteiriça migram entre os países para buscar cuidado de saúde. Estudo realizado por pesquisadores brasileiros expõe que as gestantes oriundas do Paraguai referem buscar atendimento em Foz de Iguaçu, em virtude da gratuidade que o SUS possibilita, como também, por causa da precariedade de recursos físicos e materiais do serviço paraguaio, porém, muitas gestantes não dispõem de condições financeiras para o deslocamento mensal, acarretando em acompanhamento inadequado do pré-natal.

No presente estudo observou-se que as mulheres na regional de fronteira iniciaram o pré-natal tardiamente, apresentaram menores chances de realizarem ao menos uma ultrassonografia obstétrica em cada trimestre de gestação e morfológica, realizar os exames laboratoriais, coleta de citologia oncótica no pré-natal ou em período menor que um ano antes da gravidez, consultar-se com dentista durante a gestação, ter esquema de vacinação completo da anti-hepatite B. Esses indicadores ruins podem ser reflexo do comportamento itinerante das mulheres "brasiguaias", que procuram o serviço em fase avançada da gestação, além de não disporem de condições financeiras para o deslocamento mensal, até a cidade brasileira, interrompendo a assistência preconizada pela Rede ${ }^{9,10}$.

Os indicadores ruins na $9^{a} \mathrm{RS}$ também podem ser reflexo da crise política ocorrida nos anos 2016 a 2018 na cidade de Foz de Iguaçu, por crimes de fraudes nas licitações municipais para contratação de serviços na área da saúde. Tal acontecimento repercutiu com a diminuição de "exames de diagnósticos" em unidades de saúde do município, culminando na baixa efetividade da assistência, repercutindo graves consequências aos usuários ${ }^{30}$.

As mulheres atendidas na $17^{\mathrm{a}} \mathrm{RS}$ foram mais diagnosticadas com alguma doença durante a gestação, esse dado pode ser resultado do maior acompanhamento pré-natal, visto que o mesmo não ocorreu na $9^{a}$ RS. Neste estudo a saúde materno-infantil em região fronteiriça foi prejudicada e o acompanhamento pré-natal não foi efetivo, sendo que, a crise política não deveria interferir na assistência de saúde, nem no atendimento preconizado pela Rede Mãe Paranaense, visto que as consequências aos usuários não 
podem ser reparadas e isso pode elevar os índices de morbimortalidade materno-infantil.

\section{CONCLUSÃO}

Verificou-se divergências na assistência de pré-natal prestada aos extremos de idade materna, como também entre as RS foram identificadas diferenças significativas.

A regional fronteiriça apresentou dificuldade em realizar acompanhamento efetivo de pré-natal em suas gestantes, possivelmente, devido comportamento itinerante das muIheres da fronteira, como também por causa da crise política ocorrida nessa região, que afetou criticamente a assistência à saúde naquele período. Ressaltando que não deveria existir interferência na assistência de saúde, visto que as consequências podem elevar os índices de morbimortalidade materno-infantil.

Ambos os grupos etários e a RS fronteiriça, merecem atenção dos serviços assistenciais de saúde, principalmente para cumprimento das políticas públicas e elementos preconizados pela Rede, a fim de diminuir os riscos inerentes no acompanhamento inadequado do cuidado pré-natal.

Ressalta-se que mais estudos comparando os extremos de idade materna e saúde na região de fronteira são necessários a fim de aumentar as evidências na literatura, fornecer subsídio para planejamento de estratégias e, melhorias na assistência de saúde a esses grupos etários e população fronteiriça.

\section{REFERÊNCIAS}

1. Brasil. Ministério da Saúde. Secretaria de Atenção à Saúde - departamento de ações programáticas estratégicas. Gestação de alto risco: manual técnico. 5. ed. Brasília: Ministério da Saúde [Internet]. 2012 [acesso em 2020 jul. 20]; Disponível em: http://bvsms.saude.gov.br/bvs/publicacoes/manual_tecnico_gestacao_alto_risco.pdf

2. Davim RMB, Davim MVC. Estudo reflexivo sobre aspectos biológicos, psicossociais e atendimento pré-natal durante a gravidez na adolescência. Rev enferm UFPE [Internet]. 2016 [acesso em 2020 jul. 14]; 10(8):3108-3118. Disponível em: https://doi.org/10.5205/reuol.9373-82134-1-RV1008201639

3. Laopaiboon M, Lumbiganon P, Intarut N, Mori R, Ganchimeg T, Vogel JP, Souza JP, Gülmezoglu AM, on behalf of the WHO Multicountry Survey on Maternal Newborn Health Research Network. Advanced maternal age and pregnancy outcomes: a multicountry assessment. BJOG [Internet]. 2014 [acesso em 2020 jun. 14]; 121(Supl.1):49-56. Disponível em: https://doi.org/10.1111/1471-0528.12659

4. Brasil. Ministério da saúde. Portaria $n^{\circ} 1.459$, de 24 de junho de 2011. Institui, no âmbito do sistema único de saúde (SUS), a rede cegonha. Diário oficial da união n¹21. [Internet]. Brasília, 27 jul. 2011. [acesso em 2020 jun. 14]; Disponível em: http://bvsms.saude.gov.br/bvs/saudelegis/gm/2011/prt1459_24_06_2011.html

5. Paraná. Secretaria de Estado da Saúde do Paraná. Programa Rede Mãe Paranaense. Linha guia. Curitiba: SESA-PR [Internet]. 2018 [acesso em 2020 mai. 20]; Disponível em: http://www.saude.pr.gov.br/ arquivos/File/LinhaGuiaMaeParanaense_2018.pdf

6. Kahveci B, Melekoglu R, Evruke IC, Cetin C. The effect of advanced maternal age on perinatal outcomes in nulliparous singleton pregnancies. BMC Pregnancy and Childbirth [Internet]. 2018 [acesso em 2020 set. 03]; 18(343). Disponível em: https://doi.org/10.1186/s12884-018-1984-x 
7. Viellas EF, Domingues RMSM, Dias MAB, Gama SGN, Theme MMF, Costa JV, Bastos MH, Leal MC. Assistência pré-natal no Brasil. Cad Saúde Pública [Internet]. 2014 [acesso em 2019 ago. 03]; 30(Supl.1):S85-S100. Disponível em: http://dx.doi.org/10.1590/0102-311X00126013

8. Albuquerque JL. Conflito e integração nas fronteiras dos "brasiguaios". Cad CRH [Internet]. 2010 [acesso em 2019 dez. 14]; 23(60):579-590. Disponível em: http://dx.doi.org/10.1590/S0103-49792010000300009

9. Mello F, Victora CG, Gonçalves H. Saúde nas fronteiras: análise quantitativa e qualitativa da clientela do Centro Materno Infantil de Foz do Iguaçu, Brasil. Cien Saúde colet [Internet]. 2015 [acesso em 2019 set. 17]; 20(7):2135-2145. Disponível em: http://dx.doi.org/10.1590/1413-81232015207.09462014

10. Zaslavsky R, Goulart BNG. Migração pendular e atenção à saúde na região de fronteira. Cien Saúde colet [Internet]. 2017 [acesso em 2019 set. 20]; 22(12):3981-3986. Disponível em: http://dx.doi. org/10.1590/1413-812320172212.03522016

11. Paraná. Secretaria de Estado da Saúde do Paraná. Plano Estadual de Saúde Paraná 2016-2019. Curitiba: SESA-PR [Internet]. 2016 [acesso em 2021 jun. 08]. Disponível em: https://www.saude.pr.gov.br/sites/ default/arquivos_restritos/files/documento/2020-03/planoestadualsaude_2016.pdf

12. Marino JL, Lewis LN, Bateson D, Hickey M, Skinner SR. Teenage mothers. Aust Fam Physician [Internet]. 2016 [acesso em 2020 jun. 04]; 45(10):712-717. Disponível em: https://www.racgp.org.au/afp/2016/ october/teenage-mothers/

13. Leftwich HK, Alves MVO. Adolescent Pregnancy. Pediatr Clin North Am. [Internet]. 2017 [acesso em 2020 jun. 04]; 64(2):381-388. Disponível em: https://doi.org/10.1016/j.pcl.2016.11.007

14. Sauer MV. Reproduction at an advanced maternal age and maternal health. Fertility and Sterilily [Internet]. 2015 [acesso em 2020 jul. 24]; 103(5):1136-1143. Disponível em: https://doi.org/10.1016/j.fertnstert.2015.03.004

15. Traisrisilp K, Tongsong T. Pregnancy outcomes of mothers with very advanced maternal age (40 years or more). J Med Assoc Thail 2015; 98(2):117-122.

16. Jatlaoui TC, Boutot ME, Mandell MG, Whiteman MK, Ti A, Petersen E, Pazol K. Abortion Surveillance - United States, 2015. MMWR Surveill Summ [Internet]. 2018 [acesso em 2019 dez. 05]; 67(13):1-45. Disponível em: https://www.cdc.gov/mmwr/volumes/67/ss/ss6713a1.htm

17. World Health Organization. The World health report 2005: make every mother and child count. Geneva: WHO [Internet]. 2005 [acesso em 2020 jul. 06]; Disponível em: http://www.who.int/whr/2005/whr2005_ en.pdf?ua=1

18. Neal S, Mahendra S, Bose K, Camacho AV, Mathai M, Nove A, Santana F, Matthews Z. The causes of maternal mortality in adolescents in low and middle income countries: a systematic review of the literature. BMC Pregnancy and Childbirth [Internet]. 2016 [acesso em 2020 jul. 06]; 16(352):1-18. Disponível em: https://bmcpregnancychildbirth.biomedcentral.com/articles/10.1186/s12884-016-1120-8

19. Maravilla JC, Betts KS, Cruz CC, Alati R. Factors influencing repeated teenage pregnancy: a review and meta-analysis. Am J Obstet Gynecol [Internet]. 2017 [acesso em 2020 jul. 06]; 217(5):527-545. Disponível em: https://linkinghub.elsevier.com/retrieve/pii/S0002937817305227

20. Galvão RBF, Figueira CO, Borovac-Pinheiro A, Paulino DSM, Faria-Schützer DB, Surita FG. Hazards of Repeat Pregnancy during Adolescence: A Case-control Study, Rev Bras Ginecol Obstet [Internet]. 2018 [acesso em 2019 ago. 14]; 40(8):437-443. Disponível em: <https://www.thieme-connect.com/DOI/ DOI?10.1055/s-0038-1666811

21. Carloto DR, Gil filho SF. O espaço de representação da comunidade árabe-muçulmana de Foz do Iguaçu - Paraná. In: Anais do II Colóquio Nacional do NEER - Núcleo de Estudos em Espaços e Representações; 2007 dez 05-07; Salvado, Brasil. Salvador: UFBA; 2007. [acesso em 2019 dez. 03]. Disponível em: http://www.neer.com.br/anais/NEER-2/autor_d.html

22. Marquetti MGK. Análise da influência das redes sociais no consumo de narguilé por adolescentes residentes em Foz do Iguaçu-PR [dissertação] [Internet]. Foz do Iguaçu: BDTD Unioeste; 2017. [acesso em 2019 dez. 03]. Disponível em: http://tede.unioeste.br/handle/tede/2930 
23. Menezes AHR, Dalmas JC, Scarinci IC, Maciel SM, Cardelli AAM. Fatores associados ao uso regular de cigarros por -adolescentes estudantes de escolas públicas de Londrina, Paraná, Brasil. Cad Saúde Pública [Internet]. 2014 [acesso em 2019 nov. 19]; 30(4):774-784. Disponível em: http://dx.doi.org/10.1590/ 0102-311X00173412

24. Canhaço EE, Bergamo AM, Lippi UG, Lopes RGC. Perinatal outcomes in women over 40 years of age compared to those of other gestations. Einstein [Internet]. 2015 [acesso em 2019 ago. 01]; 13(1):58-64. Disponível em: http://dx.doi.org/10.1590/S1679-45082015AO3204

25. Brosens I, Muter J, Gargett CE, Puttemans P, Benagiano G, Brosens JJ. The impact of uterine immaturity on obstetrical syndromes during adolescence. Am J Obstet Gynecol [Internet]. 2017 [acesso em 2020 jun. 04]; 217(5):546-55. Disponível em: https://www.ajog.org/article/S0002-9378(17)30688-9/fulltext

26. Kirbas A, Gulerman HC, Daglar K. Pregnancy in Adolescence: Is It an Obstetrical Risk? J Pediatric Adolesc Gynecol [Internet]. 2016 [acesso em 2019 ago. 01]; 29(4):367-371. Disponível em: https://doi. org/10.1016/j.jpag.2015.12.010

27. Santos NLAC, Costa COM, Amaral MTR, Vieira GO, Bacelar EB, Almeida AHV. Gravidez na adolescência: análise de fatores de risco para baixo peso, prematuridade e cesariana. Cien saúde colet [Internet]. 2014 [acesso em 2019 ago. 01]; 19(3):719-726. Disponível em: http://dx.doi.org/10.1590/141381232014193.18352013

28. Oliveira LL, Gonçalves AC, Costa JSD, Bonilha ALL. Fatores maternos e neonatais relacionados à prematuridade. Rev Esc Enferm USP [Internet]. 2016 [acesso em 2020 jul. 22]; 50(3):382-389. Disponível em: http://dx.doi.org/10.1590/S0080-623420160000400002

29. Han SN, Verheecke M, Vandenbroucke T, Gziri MM, Calsteren KV, Amant F. Management of gynecological cancers during pregnancy. Curr Oncol Rep [Internet]. 2014 [acesso em 2019 set. 02]; 16(415):1-10. Disponível em: https://doi.org/10.1007/s11912-014-0415-z

30. Foz do Iguaçu. Resolução $n^{\circ}$ 09/2017, de 31 de março de 2017. Não aprova o Relatório Anual de Gestão-RAG/2016, da Secretaria Municipal da Saúde de Foz do Iguaçu/PR. Diário Oficial do Município ${ }^{\circ}$ 3.03803 de abril de 2017; Seção 1:33-35. [Internet]. 2017 [acesso em 2019 dez. 03]; Disponível em: http:// www.pmfi.pr.gov.br/ArquivosDB;jsessionid=4b106df7f114f74592a80f8db4dc?idMidia=100928

RECEBIDO: 19/11/2020

ACEITO: $21 / 06 / 2021$ 